\title{
PARTIAL COLORING, VERTEX DECOMPOSABILITY, AND SEQUENTIALLY COHEN-MACAULAY SIMPLICIAL COMPLEXES
}

\author{
JENNIFER BIERMANN, CHRISTOPHER A. FRANCISCO, \\ HUY TÀI HÀ AND ADAM VAN TUYL
}

\begin{abstract}
In attempting to understand how combinatorial modifications alter algebraic properties of monomial ideals, several authors have investigated the process of adding "whiskers" to graphs. In this paper, we study a similar construction for building a simplicial complex $\Delta_{\chi}$ from a coloring $\chi$ of a subset of the vertices of $\Delta$ and give necessary and sufficient conditions for this construction to produce vertex decomposable simplicial complexes. We apply this work to strengthen and give new proofs about sequentially CohenMacaulay edge ideals of graphs.
\end{abstract}

1. Introduction. Square-free monomial ideals are intimately connected to combinatorics. This connection raises the natural question: how do changes in combinatorial structures affect algebraic properties of associated square-free monomial ideals? In [15], Villarreal investigates the process of adding whiskers to a finite simple graph, and (citing also Fröberg, Herzog and Vasconcelos) proves that the edge ideal of a graph with whiskers added to every vertex is always Cohen-Macaulay. To add a whisker to a vertex, one adds an additional vertex and an edge between the old vertex and the new one.

Generalizing Villarreal's work, in [8] the second and the third authors studied additions of whiskers to subsets of the vertices that produce sequentially Cohen-Macaulay edge ideals. The configuration of the whiskers, not the number, determines when the resulting ideals are sequentially Cohen-Macaulay, demonstrating the subtlety of the

2010 AMS Mathematics subject classification. Primary 05A15, 05E45, 13 F55.

Keywords and phrases. Simplicial complex, vertex decomposable, whiskers, sequentially Cohen-Macaulay.

The second author is partially supported by a grant from the Simons Foundation, grant No. 199124. The third author is partially supported by NSA grant H9823011-1-0165. The fourth author acknowledges the support of NSERC. 2014

Received by the editors on November 3, 2013, and in revised form on April 10, 
problem. The techniques in [8] are mostly algebraic, focusing on when the cover ideals are componentwise linear, a property which is equivalent to the Alexander dual being sequentially Cohen-Macaulay.

In a different direction, several authors have used methods from combinatorial topology to study similar phenomena. The primary combinatorial object in these efforts is the independence complex of a graph, the simplicial complex whose Stanley-Reisner ideal coincides with the edge ideal of the graph. For instance, Woodroofe [16] and Dochtermann and Engström [7] use combinatorial topology to prove that the independence complex of a chordal graph is vertex decomposable, implying that the edge ideal is sequentially Cohen-Macaulay. Dochtermann and Engström [7] also show that the independence complex of a completely whiskered graph is a pure vertex decomposable simplicial complex, and consequently, Cohen-Macaulay, thus giving a combinatorial topological proof of Villarreal's result. Cook and Nagel [6] use full clique-whiskering, a technique that begins by partitioning the vertex set of a graph into cliques. For each of these cliques, one adds a new vertex and connects it to each vertex in the clique. Cook and Nagel prove that fully clique-whiskered graphs are vertex-decomposable [6, Theorem 3.3]; when the cliques in the partition each consist of a single vertex, this recovers the results of Villarreal and DochtermannEngström.

The first and fourth authors take a blended approach in [1] to extend these results about independence complexes of graphs to all simplicial complexes. Starting with any coloring $\chi$ of the vertices of $\Delta$, they construct a new simplicial complex $\Delta_{\chi}$ that is vertex decomposable. The whiskering construction of Villarreal and the clique-whiskering technique of Cook and Nagel [6] become special instances of this construction.

The constructions in $[\mathbf{1}, \mathbf{6}, \mathbf{1 5}]$ always result in pure vertex decomposable simplicial complexes (and thus, Cohen-Macaulay complexes). The algebraic results of [8] are therefore not a consequence of these results because the corresponding independence complex associated to the partially whiskered graph is not necessarily pure.

In this paper, in the spirit of [8], we extend the construction in [1] to partial whiskerings of simplicial complexes. We start with a partial coloring $\chi$ of $\Delta$ (see Definition 2.9) and use $\chi$ and $\Delta$ to build 
a new simplicial complex $\Delta_{\chi}$ (see Construction 3.1), which we call a partially whiskered simplicial complex. We give a necessary and sufficient condition for a partially whiskered simplicial complex to be vertex decomposable.

Theorem 1.1 (Theorem 3.4). Let $\Delta$ be a simplicial complex on the vertex set $V$, and let $W$ be a subset of $V$. Let $\chi$ be the s-coloring of $\left.\Delta\right|_{W}$ given by $W=W_{1} \cup \cdots \cup W_{s}$. Then $\Delta_{\chi}$ is vertex decomposable if and only if $\left.\operatorname{link}_{\Delta}(\mu)\right|_{\bar{W}}$ is vertex decomposable for every face $\mu$ of $\Delta$ such that $\mu \subseteq W$.

Theorem 3.4 has a number of consequences. Corollary 3.7 shows that when $W=V, \Delta_{\chi}$ is always vertex decomposable, thus recovering the main result of [1]. Corollary 3.7 also gives the analog to the numerical bound of the second and third authors [8] for graphs. Namely, if one has a simplicial complex with $n$ vertices, and $|W| \geq n-3$, one gets a vertex decomposable simplicial complex. As in the case of graphs, this bound is sharp (see Example 3.8).

In Section 4, we apply Theorem 3.4 to study edge ideals of graphs. In particular, we get necessary and sufficient conditions for a whiskered graph to be vertex decomposable (see Theorem 4.6). This result yields Corollary 4.8, a new proof for [8, Theorem 3.3] specifying which configurations of whiskers force the edge ideal to be sequentially CohenMacaulay; this provides the combinatorial approach to the results of [8] sought in [7]. We also use Theorem 3.4 to classify which whiskered bipartite graphs are sequentially Cohen-Macaulay (see Theorem 4.11).

Our paper is organized as follows. In Section 2, we recall the relevant background. In Section 3, we present the main theorems and derive some of their consequences. Section 4 applies our results to independence complexes of graphs.

2. Background. We recall the relevant background on simplicial complexes.

Definition 2.1. A finite simplicial complex $\Delta$ on a finite vertex set $V$ is a collection of subsets of $V$ with the property that, if $\sigma \in \Delta$ and $\tau \subseteq \sigma$, then $\tau \in \Delta$. The elements of $\Delta$ are called faces. The maximal faces of $\Delta$, with respect to inclusion, are the facets. 
The vertex sets of our simplicial complexes will be either the set $\left\{x_{1}, \ldots, x_{n}\right\}$ or the set $\left\{x_{1}, \ldots, x_{n}, y_{1}, \ldots, y_{s}\right\}$. If $\Delta$ is a simplicial complex and $\sigma \in \Delta$, then we say $\sigma$ has dimension $d$ if $|\sigma|=d+1$ (by convention, the empty set has dimension -1 ). We say $\Delta$ is pure if all of its facets have the same dimension; otherwise, $\Delta$ is non-pure. If $F_{1}, \ldots, F_{t}$ is a complete list of the facets of $\Delta$, we sometimes write $\Delta$ as $\Delta=\left\langle F_{1}, \ldots, F_{t}\right\rangle$. If $\sigma \in \Delta$ is a face, then the deletion of $\sigma$ from $\Delta$ is the simplicial complex defined by

$$
\Delta \backslash \sigma=\{\tau \in \Delta \mid \sigma \nsubseteq \tau\} .
$$

The link of $\sigma$ in $\Delta$ is the simplicial complex defined by

$$
\operatorname{link}_{\Delta}(\sigma)=\{\tau \in \Delta \mid \sigma \cap \tau=\emptyset, \sigma \cup \tau \in \Delta\} .
$$

When $\sigma=\{v\}$, we shall abuse notation and write $\Delta \backslash v$ (respectively, $\left.\operatorname{link}_{\Delta}(v)\right)$ for $\Delta \backslash\{v\}$ (respectively, $\operatorname{link}_{\Delta}(\{v\})$ ).

We shall be particularly interested in the class of vertex decomposable simplicial complexes. This class was first introduced in the pure case by Provan and Billera [13] and in the non-pure case by Björner and Wachs [3]. We recall the non-pure version.

Definition 2.2. A simplicial complex $\Delta$ on vertex set $V$ is called vertex decomposable if

(i) $\Delta$ is a simplex, or

(ii) there is some vertex $v \in V$ such that $\Delta \backslash v$ and $\operatorname{link}_{\Delta}(v)$ are vertex decomposable and do not share any facets; such a vertex $v$ is called a shedding vertex of $\Delta$.

Remark 2.3. When $\Delta$ is vertex decomposable, then $\Delta$ also inherits other combinatorial and algebraic properties. In particular, if $\Delta$ is pure and vertex decomposable, then $\Delta$ has a pure shelling, and its StanleyReisner ring $R / I_{\Delta}$ is Cohen-Macaulay. If $\Delta$ is non-pure and vertex decomposable, then $\Delta$ is still shellable, in the non-pure sense of Björner and Wachs [3], and its Stanley-Reisner $\operatorname{ring} R / I_{\Delta}$ is sequentially Cohen-Macaulay (see Section 4 for a definition). We point the reader to the text of Herzog and Hibi [11] for a complete treatment of these ideas. 
For simplicial complexes $\Delta$ and $\Omega$ over disjoint vertex sets $V$ and $U$, respectively, the join of $\Delta$ and $\Omega$, denoted by $\Delta \cdot \Omega$, is the simplicial complex over the vertex set $V \cup U$, whose faces are $\{\sigma \cup \tau \mid \sigma \in \Delta, \tau \in$ $\Omega$. Provan and Billera proved:

Theorem 2.4 ([13, Proposition 2.4]). The join $\Delta \cdot \Omega$ is vertex decomposable if and only if both $\Delta$ and $\Omega$ are vertex decomposable.

The property of vertex decomposability is preserved when taking a link. The following result was first proved in [13, Proposition 2.3] in the pure case; the non-pure case follows similarly, as noted in [12, Theorem 3.30] and [17, Proposition 3.7].

Theorem 2.5. If $\Delta$ is vertex decomposable, then $\operatorname{link}_{\Delta}(\sigma)$ is vertex decomposable for any $\sigma \in \Delta$.

An important notion for our main construction and results in Section 3 is that of a coloring of a simplicial complex.

Definition 2.6. Let $\Delta$ be a simplicial complex on the vertex set $V$ with facets $F_{1}, \ldots, F_{t}$. An $s$-coloring of $\Delta$ is a partition of the vertices $V=V_{1} \cup \cdots \cup V_{s}$ (where the sets $V_{i}$ are allowed to be empty) such that $\left|F_{i} \cap V_{j}\right| \leq 1$ for all $1 \leq i \leq t, 1 \leq j \leq s$. We will sometimes write that $\chi$ is an $s$-coloring of $\Delta$ to mean $\chi$ is a specific partition of $V$ that gives an $s$-coloring of $\Delta$. If there exists an $s$-coloring of $\Delta$, we say that $\Delta$ is $s$-colorable.

Note that the definition of an $s$-coloring is equivalent to an $s$ coloring (in the graph theoretic sense) of the 1-skeleton of the simplicial complex.

Example 2.7. If $\Delta$ is a simplicial complex on $|V|=n$ vertices, then $\Delta$ is $n$-colorable; indeed, we take our coloring to be $V=\left\{x_{1}\right\} \cup\left\{x_{2}\right\} \cup$ $\cdots \cup\left\{x_{n}\right\}$. 
In this paper, we are interested in the case in which a subset of the vertices of a simplicial complex is colored, or equivalently, in the coloring of an induced subcomplex.

Definition 2.8. Let $\Delta$ be a simplicial complex on the vertex set $V$, and let $W \subseteq V$. The restriction of $\Delta$ to $W$ (or equivalently, the induced subcomplex over $W$ ) is the subcomplex

$$
\left.\Delta\right|_{W}=\{\sigma \in \Delta \mid \sigma \subseteq W\} .
$$

Definition 2.9. Let $\Delta$ be a simplicial complex on the vertex set $V$, and let $W \subseteq V$. If $\chi$ is an $s$-coloring of the restriction $\left.\Delta\right|_{W}$, then we call $\chi$ a partial coloring of $\Delta$. We call the vertices in $W$ the colored vertices of $\Delta$ and those in $\bar{W}=V \backslash W$ the non-colored vertices.

Example 2.10. Let $\Delta=\left\langle x_{1} x_{2} x_{3}, x_{2} x_{4}\right\rangle$, and let $W=\left\{x_{1}, x_{2}, x_{4}\right\}$. Then $\left.\Delta\right|_{W}=\left\langle x_{1} x_{2}, x_{2} x_{4}\right\rangle$. Then a 2-coloring $\chi$ of $\left.\Delta\right|_{W}$ is given by $W=\left\{x_{1}, x_{4}\right\} \cup\left\{x_{2}\right\}$. So $\chi$ is a partial coloring of $\Delta$, where the vertices of $W$ are the colored vertices, and $\left\{x_{3}\right\}$ is a non-colored vertex.

3. Partial colorings and vertex decomposability. Given any simplicial complex $\Delta$ with a partial coloring $\chi$, we introduce a construction to make a new simplicial complex $\Delta_{\chi}$. Our main result gives necessary and sufficient conditions for $\Delta_{\chi}$ to be vertex decomposable. We begin with the construction of $\Delta_{\chi}$.

Construction 3.1. Let $\Delta$ be a simplicial complex on the vertex set $V=\left\{x_{1}, \ldots, x_{n}\right\}$, and let $W$ be a subset of $V$. Let $\chi$ be an s-coloring of $\left.\Delta\right|_{W}$ given by $W=W_{1} \cup \cdots \cup W_{s}$. Define $\Delta_{\chi}$ to be the simplicial complex on the vertex set $\left\{x_{1}, \ldots, x_{n}, y_{1}, \ldots, y_{s}\right\}$ with faces $\sigma \cup \tau$, where $\sigma$ is a face of $\Delta$, and $\tau$ is any subset of $\left\{y_{1}, \ldots, y_{s}\right\}$ such that, for all $y_{j} \in \tau$, we have $\sigma \cap W_{j}=\emptyset$. Note, in particular, that since $\emptyset$ is a face of $\Delta,\left\{y_{1}, y_{2}, \ldots, y_{s}\right\}$ is always a face of $\Delta_{\chi}$.

The first and fourth authors recently studied Construction 3.1 in [1] in the case that $W=V$; this case also appears in [9] and implicitly in a proof in [2]. We call the process of adding a new vertex $y_{i}$ for a color class $W_{i}$ whiskering, and the resulting complex $\Delta_{\chi}$ the (partially) whiskered simplicial complex. 
Example 3.2. Consider the simplicial complex $\Delta=\left\langle x_{1} x_{2} x_{3}, x_{2} x_{4}\right\rangle$ of Example 2.10. If $W=\left\{x_{1}, x_{2}, x_{4}\right\}$, take $\chi$ to be the coloring of $\left.\Delta\right|_{W}$ given by $W=\left\{x_{1}, x_{4}\right\} \cup\left\{x_{2}\right\}$. Then $\Delta_{\chi}=\left\langle x_{3} y_{1} y_{2}, x_{4} y_{2}, x_{1} x_{3} y_{2}, x_{2} x_{3} y_{1}\right.$, $\left.x_{2} x_{4}, x_{1} x_{2} x_{3}\right\rangle . \Delta$ and $\Delta_{\chi}$ are shown in Figure 1.
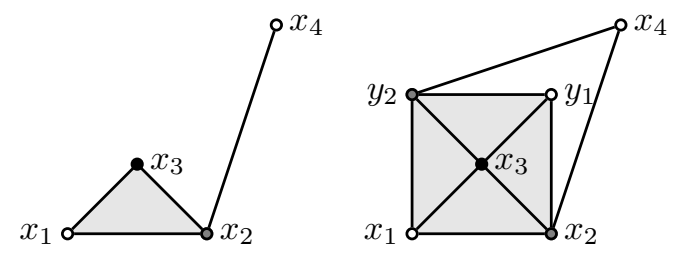

FiguRE 1. The simplicial complexes $\Delta$ (left) and $\Delta_{\chi}$ (right).

Remark 3.3. To forestall any potential confusion, "whiskering a vertex," as first defined in [15], referred to adding a new additional vertex to a graph and joining the new and old vertices by an edge. This operation was defined in terms of the finite simple graph. However, this procedure also results in a change in the independence complex of the graph (see Section 4 for details). In our definition, when we use the term "whiskering," we are generalizing the operation that changes the independence complex, not the graph.

Our main result is necessary and sufficient conditions for $\Delta_{\chi}$ to be vertex decomposable.

Theorem 3.4. Let $\Delta$ be a simplicial complex on the vertex set $V$, and let $W$ be a subset of $V$. Let $\chi$ be the s-coloring of $\left.\Delta\right|_{W}$ given by $W=W_{1} \cup \cdots \cup W_{s}$. Then $\Delta_{\chi}$ is vertex decomposable if and only if $\left.\operatorname{link}_{\Delta}(\mu)\right|_{\bar{W}}$ is vertex decomposable for every face $\mu$ of $\Delta$ such that $\mu \subseteq W$.

Remark 3.5. The special case $\mu=\emptyset$ in Theorem 3.4 is instructive. Because $\operatorname{link}_{\Delta}(\emptyset)=\Delta$, the link hypothesis imposes the condition that $\left.\Delta\right|_{\bar{W}}$ is vertex decomposable.

Proof of Theorem 3.4. $(\Leftarrow)$. We proceed by induction on the number of vertices of $\Delta$. The base case is the empty simplicial complex $\Delta=$ 
$\{\emptyset\}$. In this case, the only partial coloring of $\Delta$ is $W=W_{1} \cup \cdots \cup W_{s}$, where all the $W_{i}$ are empty. Then $\Delta_{\chi}$ is the simplex $\left\langle\left\{y_{1}, \ldots, y_{s}\right\}\right\rangle$, which is vertex decomposable.

Now let $\Delta$ be a simplicial complex on vertex set $V=\left\{x_{1}, \ldots, x_{n}\right\}$, and let $W \subseteq V$. If $W=\emptyset$, then $\Delta_{\chi}=\Delta \cdot\left\langle\left\{y_{1}, \ldots, y_{s}\right\}\right\rangle$, the join of two simplicial complexes. The link hypothesis with $\mu=\emptyset$ implies that $\left.\Delta\right|_{\bar{\emptyset}}=\Delta$ is vertex decomposable. It then follows from Theorem 2.4 that $\Delta_{\chi}$ is vertex decomposable. We can therefore assume that $\Delta \neq\{\emptyset\}$ and $W \neq \emptyset$.

Let $w \in W$. After relabelling, we will assume that $w \in W_{1}$. To prove that $\Delta_{\chi}$ is vertex decomposable, we will show that $\Delta_{\chi} \backslash w$ and $\operatorname{link}_{\Delta_{\chi}}(w)$ are both vertex decomposable.

Recall that the faces of $\Delta_{\chi}$ are of the form $\sigma \cup \tau$, where $\sigma$ is a face of $\Delta$ and $\tau \subseteq\left\{y_{1}, \ldots y_{s}\right\}$ such that, if $y_{j} \in \tau$, then $W_{j} \cap \sigma=\emptyset$. Note that

$$
\begin{aligned}
\Delta_{\chi} \backslash w & =\left\{\sigma \cup \tau \in \Delta_{\chi} \mid w \notin \sigma \cup \tau\right\} \\
& =\left\{\sigma \cup \tau \in \Delta_{\chi} \mid w \notin \sigma\right\} \\
& =(\Delta \backslash w)_{\chi^{\prime}},
\end{aligned}
$$

where $\chi^{\prime}$ is the partial coloring $\left(W_{1} \backslash\{w\}\right) \cup W_{2} \cup \cdots \cup W_{s}$ of $\Delta \backslash w$ induced by the coloring $\chi$ of $\Delta$. Since $w \in W$, the uncolored vertices of $\Delta$ and $\Delta \backslash w$ are the same set $\bar{W}$. Now let $\mu$ be a face of $\Delta \backslash w$ such that $\mu \subseteq(W \backslash\{w\})$. Then, since $w \notin \bar{W}$,

$$
\begin{aligned}
\left.\left(\operatorname{link}_{\Delta \backslash w}(\mu)\right)\right|_{\bar{W}} & =\left.\{\sigma \in(\Delta \backslash w) \mid \mu \cap \sigma=\emptyset, \mu \cup \sigma \in(\Delta \backslash w)\}\right|_{\bar{W}} \\
& =\left.\{\sigma \in \Delta \mid w \notin \sigma, \mu \cap \sigma=\emptyset, \mu \cup \sigma \in(\Delta \backslash w)\}\right|_{\bar{W}} \\
& =\left.\{\sigma \in \Delta \mid \mu \cap \sigma=\emptyset, \mu \cup \sigma \in \Delta\}\right|_{\bar{W}} \\
& =\left.\left(\operatorname{link}_{\Delta}(\mu)\right)\right|_{\bar{W}} .
\end{aligned}
$$

Thus, we have that $\left.\left(\operatorname{link}_{\Delta \backslash w}(\mu)\right)\right|_{\bar{W}}$ is vertex decomposable by hypothesis. Therefore, since $\Delta \backslash w$ is a simplicial complex on fewer than $n$ vertices with $\chi^{\prime}$ a partial coloring on $W \backslash\{w\}$ such that $\left.\left(\operatorname{link}_{\Delta \backslash w}(\mu)\right)\right|_{\bar{W}}$ is vertex decomposable for all $\mu \subseteq(W \backslash\{w\})$, induction implies that $\Delta_{\chi} \backslash w=(\Delta \backslash w)_{\chi^{\prime}}$ is vertex decomposable.

Now consider $\operatorname{link}_{\Delta_{\chi}}(w)$. We have

$$
\operatorname{link}_{\Delta_{\chi}}(w)=\left\{\sigma \cup \tau \in \Delta_{\chi} \mid w \notin \sigma \cup \tau, \sigma \cup \tau \cup\{w\} \in \Delta_{\chi}\right\}
$$




$$
\begin{aligned}
& =\left\{\sigma \cup \tau \in \Delta_{\chi} \mid w \notin \sigma,(\sigma \cup\{w\}) \cup \tau \in \Delta_{\chi}\right\} \\
& =\left(\operatorname{link}_{\Delta}(w)\right)_{\chi^{\prime \prime}}
\end{aligned}
$$

where $\chi^{\prime \prime}$ is the partial coloring of $\operatorname{link}_{\Delta}(w)$ given by $U_{2} \cup \cdots \cup U_{s}$, and $U_{j}=W_{j} \cap\left\{\right.$ vertices of $\left.\operatorname{link}_{\Delta}(w)\right\}$. Because $w \in W_{1}$, we need only consider $U_{j}$ for $j=2, \ldots, s$. Set $U=U_{2} \cup \cdots \cup U_{s}$ (i.e., the colored vertices of $\left.\operatorname{link}_{\Delta}(w)\right)$ and $\bar{U}$ to be the set of non-colored vertices of $\operatorname{link}_{\Delta}(w)$.

Note that $\bar{U}=\bar{W} \cap\left\{\right.$ vertices of $\left.\operatorname{link}_{\Delta}(w)\right\}$. Let $\mu$ be a face of $\operatorname{link}_{\Delta}(w)$ such that $\mu \subseteq U$. Since $\mu \in \operatorname{link}_{\Delta}(w)$, we have $\mu \cup\{w\} \in \Delta$. Then

$$
\operatorname{link}_{\operatorname{link}_{\Delta}(w)}(\mu)=\operatorname{link}_{\Delta}(\mu \cup\{w\}),
$$

so

$$
\left.\left(\operatorname{link}_{\operatorname{link}_{\Delta}(w)}(\mu)\right)\right|_{\bar{U}}=\left.\left(\operatorname{link}_{\operatorname{link}_{\Delta}(w)}(\mu)\right)\right|_{\bar{W}}=\left.\left(\operatorname{link}_{\Delta}(\mu \cup\{w\})\right)\right|_{\bar{W}} .
$$

By the assumption on $\Delta$, $\left.\left(\operatorname{link}_{\Delta}(\mu \cup\{w\})\right)\right|_{\bar{W}}$ is vertex decomposable. Because $\operatorname{link}_{\Delta_{\chi}}(w)=\left(\operatorname{link}_{\Delta}(w)\right)_{\chi^{\prime \prime}}$, and $\operatorname{link}_{\Delta}(w)$ is a simplicial complex on fewer than $n$ vertices, $\operatorname{link}_{\Delta_{\chi}}(w)$ is vertex decomposable by induction.

To show that $\Delta_{\chi}$ is vertex decomposable, all that remains is to show that no facet of $\operatorname{link}_{\Delta_{\chi}}(w)$ is a facet of $\Delta_{\chi} \backslash w$. Let $\sigma \cup \tau$ be a facet of $\operatorname{link}_{\Delta_{\chi}}(w)$. Then $(\sigma \cup\{w\}) \cup \tau$ is a face of $\Delta_{\chi}$, so $y_{1} \notin \tau$. On the other hand, if $\sigma \cup \tau \in \Delta_{\chi} \backslash w$, then since $\sigma \cap W_{1}=\emptyset, \sigma \cup \tau \cup\left\{y_{1}\right\}$ is also a face of $\Delta_{\chi} \backslash w$ and so the link and deletion do not share any facets.

$(\Rightarrow)$. Let $\mu \in \Delta$ be a face such that $\mu \subseteq W$, and hence $\left.\mu \in \Delta\right|_{W}$. Because $\chi$ is an $s$-coloring of $\left.\Delta\right|_{W}$, we have $\left|\mu \cap W_{i}\right| \leq 1$ for $i=1, \ldots, s$. After relabelling the $W_{j}$ 's, we may assume that $\left|\mu \cap W_{i}\right|=1$ for $i=1, \ldots, t$, and $\left|\mu \cap W_{i}\right|=0$ for $i=t+1, \ldots, s$.

By Construction 3.1, we have $\mu \cup\left\{y_{t+1}, \ldots, y_{s}\right\} \in \Delta_{\chi}$. We now claim that

$$
\left.\operatorname{link}_{\Delta}(\mu)\right|_{\bar{W}}=\operatorname{link}_{\Delta_{\chi}}\left(\mu \cup\left\{y_{t+1}, \ldots, y_{s}\right\}\right) .
$$

Notice that our conclusion will then follow from this claim and Theorem 2.5 because $\Delta_{\chi}$ is assumed to be vertex decomposable.

For any $\left.\tau \in \operatorname{link}_{\Delta}(\mu)\right|_{\bar{W}}$, we have $\tau \cup \mu \in \Delta, \tau \cap \mu=\emptyset$, and $\tau \cap W=\emptyset$. By Construction 3.1, $\tau \cup \mu \cup\left\{y_{t+1}, \ldots, y_{s}\right\} \in \Delta_{\chi}$ because $(\tau \cup \mu) \cap W_{i}=\emptyset$ 
for $i=t+1, \ldots, s$. Moreover, since $\tau \cap\left(\mu \cup\left\{y_{t+1}, \ldots, y_{s}\right\}\right)=\emptyset$, we have $\tau \in \operatorname{link}_{\Delta_{\chi}}\left(\mu \cup\left\{y_{t+1}, \ldots, y_{s}\right\}\right)$.

We now consider the reverse inclusion. Let $\tau \in \operatorname{link}_{\Delta_{x}}(\mu \cup$ $\left.\left\{y_{t+1}, \ldots, y_{s}\right\}\right)$. Thus, $\tau \cup \mu \cup\left\{y_{t+1}, \ldots, y_{s}\right\} \in \Delta_{\chi}$ and $\tau \cap(\mu \cup$ $\left.\left\{y_{t+1}, \ldots, y_{s}\right\}\right)=\emptyset$. Since $\left|\mu \cap W_{i}\right|=1$ for $i=1, \ldots, t$ we know that $y_{i} \notin \tau$ for $i=1, \ldots, t$. Therefore, $\tau \subseteq\left\{x_{1}, \ldots, x_{n}\right\}$, and $\tau \cup \mu$ must be a face of $\Delta$. Thus, $\tau \in \operatorname{link}_{\Delta}(\mu)$.

Further, since $\tau \cup \mu \in \Delta$ and $\left|\mu \cap W_{i}\right|=1$ for $i=1, \ldots, t$, we have $\left|\tau \cap W_{i}\right|=0$ for $i=1, \ldots, t$. Since $\tau \cup \mu \cup\left\{y_{t+1}, \ldots, y_{s}\right\} \in \Delta_{\chi}$, we have $\left|\tau \cap W_{i}\right|=0$ for $i=t+1, \ldots, s$ as well. Therefore, $\left.\tau \in \operatorname{link}_{\Delta}(\mu)\right|_{\bar{W}}$.

Remark 3.6. Let $\Delta=\left\langle x_{1} x_{2} x_{3} x_{4}, x_{1} x_{3} x_{4} x_{5}, x_{1} x_{3} x_{5} x_{6}, x_{1} x_{2} x_{5} x_{6}\right.$, $\left.x_{2} x_{3} x_{6}\right\rangle$, and let $\chi$ be the coloring given by $W=\left\{x_{1}\right\} \cup\left\{x_{2}\right\}$. Then $\left.\Delta\right|_{\bar{W}}$, $\left.\operatorname{link}_{\Delta}\left(x_{1}\right)\right|_{\bar{W}}$, and $\left.\operatorname{link}_{\Delta}\left(x_{2}\right)\right|_{\bar{W}}$ are all vertex decomposable. However, $\left.\operatorname{link}_{\Delta}\left(\left\{x_{1}, x_{2}\right\}\right)\right|_{\bar{W}}$ is not vertex decomposable, so by Theorem 3.4, neither is $\Delta_{\chi}$.

We now give a bound on the number of vertices to color to ensure that $\Delta_{\chi}$ is vertex decomposable. The following corollary also recovers [1, Theorem 3.7] in the case where $|V \backslash W|=0$.

Corollary 3.7. Let $\Delta$ be a simplicial complex on vertex set $V, W$ a subset of $V$ and $\chi$ a coloring of $\left.\Delta\right|_{W}$. If $|V \backslash W| \leq 3$, then $\Delta_{\chi}$ is vertex decomposable.

Proof. All simplicial complexes on three or fewer vertices are vertex decomposable. Since $|\bar{W}|=|V \backslash W| \leq 3,\left.\operatorname{link}_{\Delta}(\mu)\right|_{\bar{W}}$ is vertex decomposable for any $\mu \in \Delta$ such that $\mu \subseteq W$. Thus, by Theorem 3.4, $\Delta_{\chi}$ is vertex decomposable.

The previous corollary is an analog of a bound of the second and third authors [8, Corollary 3.5]. The numerical bound on the cardinality of $\bar{W}$ in Corollary 3.7 is sharp:

Example 3.8. Let $\Delta=\left\langle x_{1} x_{2} x_{3}, x_{3} x_{4} x_{5}\right\rangle, W=\left\{x_{3}\right\}$ and $\chi$ be the coloring of $\left.\Delta\right|_{W}$ given by $W=W_{1}=\left\{x_{3}\right\}$, so $|\bar{W}|=4$. Then $\operatorname{link}_{\Delta}(\emptyset)=\left.\Delta\right|_{\bar{W}}=\left\langle x_{1} x_{2}, x_{4} x_{5}\right\rangle$, which is not vertex decomposable. Thus, $\Delta$ with this coloring does not fit the conditions of Theorem 3.4. 
Indeed, $\Delta_{\chi}=\left\langle x_{4} x_{5} y_{1}, x_{1} x_{2} y_{1}, x_{3} x_{4} x_{5}, x_{1} x_{2} x_{3}\right\rangle$ is not vertex decomposable.

As noted in [8], it is not necessarily the number of whiskers but rather their configuration that determines whether the resulting ideal is sequentially Cohen-Macaulay. A similar phenomenon occurs in our setting. In particular, given any coloring of $\Delta$, if $\chi$ is its restriction to all but one color class, then $\Delta_{\chi}$ is vertex decomposable.

Corollary 3.9. Let $\Delta$ be a simplicial complex on the vertex set $V=$ $\left\{x_{1}, \ldots, x_{n}\right\}$, and let $\chi$ be an s-coloring of $\Delta$ given by $V=V_{1} \cup \cdots \cup V_{s}$. For each $i=1, \ldots, s$, let $\chi_{i}$ be the induced partial coloring of $\left.\Delta\right|_{Y_{i}}$ given by $Y_{i}=V_{1} \cup \cdots V_{i-1} \cup V_{i+1} \cup \cdots V_{s}$. Then $\Delta_{\chi_{i}}$ is vertex decomposable for each $i=1, \ldots, s$.

Proof. It suffices to prove the statement for $i=1$. Let $Y_{1}=$ $V_{2} \cup \cdots \cup V_{s}$ be the induced partial coloring of $\Delta$ given by $\chi_{1}$. Then $\overline{Y_{1}}=V_{1}$. Since $\chi$ is a coloring, if $\sigma \in \Delta$ and $\sigma \subseteq V_{1}$, then $|\sigma| \leq 1$. Then, for any $\mu \subseteq Y$, $\left.\operatorname{link}_{\Delta}(\mu)\right|_{V_{1}}$ is either the simplicial complex $\{\emptyset\}$ or a zero-dimensional simplex. Because these simplicial complexes are vertex decomposable, Theorem 3.4 implies the desired result.

4. Sequentially Cohen-Macaulay edge ideals. We round out this paper by applying Theorem 3.4 to edge ideals of graphs. In particular, we give a new proof of [8, Theorem 3.3] and classify when a whiskered bipartite graph is sequentially Cohen-Macaulay.

We recall some terminology. Let $G=(V, E)$ be a finite simple graph. We say $W \subseteq V$ is an independent set if for all $e \in E, e \cap W \neq e$. We can form a simplicial complex from the independent sets of $G$ :

Definition 4.1. Let $G$ be a finite simple graph. The independence complex of $G$, denoted Ind $(G)$, is the simplicial complex $\operatorname{Ind}(G)=$ $\{W \mid W$ is an independent set of $G\}$.

If $V=\left\{x_{1}, \ldots, x_{n}\right\}$, we can identify the vertices of $G$ with the variables of $R=k\left[x_{1}, \ldots, x_{n}\right]$. The edge ideal of $G$, denoted $I(G)$, is the quadratic squarefree monomial ideal generated by the monomials 
$x_{i} x_{j}$ whenever $\left\{x_{i}, x_{j}\right\} \in E$. The edge ideal of $G$ is the Stanley-Reisner ideal of $\operatorname{Ind}(G)$, i.e., $I(G)=I_{\operatorname{Ind}(G)}$.

We recall the definition of sequentially Cohen-Macaulay modules as it pertains to $I(G)$.

Definition 4.2. The graph $G$ is sequentially Cohen-Macaulay if $R / I(G)$ is sequentially Cohen-Macaulay; that is, there exists a finite filtration of graded $R$ modules $0=M_{0} \subset M_{1} \subset \cdots \subset M_{t}=R / I(G)$ such that $M_{i} / M_{i-1}$ is Cohen-Macaulay for each $i \in\{1, \ldots, t\}$, and for all $i \in\{1, l \ldots, t-1\}, \operatorname{dim} M_{i} / M_{i-1}<\operatorname{dim} M_{i+1} / M_{i}$. The ideal $I(G)$ is a sequentially Cohen-Macaulay edge ideal if $G$ is sequentially Cohen-Macaulay.

If we specialize the general theory of vertex decomposable simplicial complexes, we have the following link between these objects.

Theorem 4.3. If $\operatorname{Ind}(G)$ is vertex decomposable, then $G$ is sequentially Cohen-Macaulay.

Given a subset $S \subseteq V$, we denote by $G \cup W(S)$ the graph obtained by adding whiskers to all the vertices of $S$. After relabelling, we can always assume $S=\left\{x_{1}, \ldots, x_{s}\right\}$. The following lemma, which is simply applying the definitions, describes the connection between the whiskered graph $G \cup W(S)$ and Construction 3.1.

Lemma 4.4. Let $G$ be a finite simple graph on the vertex set $V$, and let $S \subseteq V$. Let $\chi$ be the s-coloring of $\left.\operatorname{Ind}(G)\right|_{S}$ given by $S=$ $\left\{x_{1}\right\} \cup \cdots \cup\left\{x_{s}\right\}$. Then Ind, $(G \cup W(S))=\operatorname{Ind}(G)_{\chi}$.

Remark 4.5. One can also recover the clique-starring and cliquewhiskering techniques of Woodroofe [16] and Cook and Nagel [6] from Construction 3.1, coloring all vertices and allowing each coloring class to have more than one vertex.

When restricted to independence complexes of graphs, Theorem 3.4 gives us necessary and sufficient conditions for a whiskered graph to have vertex decomposable independence complex. Below, $G \backslash \mu$ denotes 
a graph $G$ with the vertices of $\mu$ and adjacent edges removed. For any subset $W \subseteq V$, we use $\left.G\right|_{W}$ to denote the induced subgraph of $G$ on $W$, i.e., the graph with vertices the elements of $W$ and edge set $\{e \in E \mid e \subseteq W\}$.

Theorem 4.6. Let $\operatorname{Ind}(G)$ be the independence complex of a graph $G$ on a vertex set $V$, and let $S \subseteq V$. Then $\operatorname{Ind}(G \cup W(S))$ is vertex decomposable if and only if $\operatorname{Ind}\left(\left.(G \backslash \mu)\right|_{\bar{S}}\right)$ is vertex decomposable for all $\mu \in \operatorname{Ind}(G)$ with $\mu \subseteq S$.

Proof. By Lemma 4.4, Ind $(G \cup W(S))=\operatorname{Ind}(G)_{\chi}$ for the $s$-coloring $\chi$ of Ind $(G)_{S}$ given by $S=\left\{x_{1}\right\} \cup \cdots \cup\left\{x_{s}\right\}$. On the other hand, for any $\mu \subseteq S$, one can show that

$$
\left.\operatorname{link}_{\operatorname{Ind}(G)}(\mu)\right|_{\bar{S}}=\left.\operatorname{Ind}(G \backslash \mu)\right|_{\bar{S}}=\operatorname{Ind}\left(\left.(G \backslash \mu)\right|_{\bar{S}}\right) .
$$

Thus, the statement is simply restricting Theorem 3.4 to independence complexes.

Recall that a graph $G$ is chordal if it has no induced cycles of length $\geq 4$. The independence complexes of chordal graphs are particularly nice:

Theorem 4.7 ([7, Theorem 4.1][16, Corollary 7(2)]). If $G$ is chordal graph, then Ind $(G)$ is vertex decomposable.

We now show how Theorem 4.6 not only allows us to give a new proof of [8, Theorem 3.3] but also strengthen it. The original conclusion of [8, Theorem 3.3] is that the associated edge ideals are sequentially CohenMacaulay. This now follows from Corollary 4.8 and Theorem 4.3.

Corollary 4.8. Let $G$ be a finite simple graph, and let $S \subseteq V$. Suppose that $G \backslash S$, the induced subgraph over the vertices $V \backslash S$, is either a chordal graph or the five cycle $C_{5}$. Then $\operatorname{Ind}(G \cup W(S))$ is vertex decomposable. In particular, $G \cup W(S)$ is sequentially Cohen-Macaulay.

Proof. First assume that $G \backslash S$ is a chordal graph. Let $V=$ $\left\{x_{1}, \ldots, x_{n}\right\}$ and suppose, after relabelling, $S=\left\{x_{1}, \ldots, x_{s}\right\}$. Let $\chi$ be the $s$-coloring of $\left.\operatorname{Ind}(G)\right|_{S}$ given by $S=\left\{x_{1}\right\} \cup \cdots \cup\left\{x_{s}\right\}$. For 
any $\mu \subseteq S,\left.(G \backslash \mu)\right|_{\bar{S}}$ is an induced subgraph of $\left.G\right|_{\bar{S}}$, so it is chordal. By Theorem 4.7, Ind $\left(\left.(G \backslash \mu)\right|_{S}\right)$ is vertex decomposable. Now apply Theorem 4.6.

The proof for the case $G \backslash S$ is a five-cycle is similar because the independence complex of a five-cycle is vertex decomposable, as are any induced subgraphs.

Remark 4.9. The proof of Corollary 4.8 only requires that all the induced subgraphs of $G \backslash S$ have the property that their independence complexes be vertex decomposable. Woodroofe [16] has shown that all graphs whose only induced cycles are either three-cycles or five-cycles have this property; this family contains the family of graphs listed in the above corollary. Our statement of Corollary 4.8 was chosen to highlight the connection to the work of [8].

In the case of bipartite graphs, we can strengthen our results and classify exactly when a whiskered bipartite graph is sequentially CohenMacaulay. Recall that we say that a graph $G=(V, E)$ is bipartite if there exists a partition $V=V_{1} \cup V_{2}$ such that, for all $e \in E$, we have $e \cap V_{1} \neq \emptyset$ and $e \cap V_{2} \neq \emptyset$. We then need the following result of the fourth author.

Theorem 4.10 ([14, Theorem 2.10]). Let $G$ be a bipartite graph. Then Ind $(G)$ is vertex decomposable if and only if $G$ is sequentially CohenMacaulay.

We then have the following classification.

Theorem 4.11. Let $I(G)$ be the edge ideal of a bipartite graph $G$ on a vertex set $V$ and let $S \subseteq V$. Then $G \cup W(S)$ is sequentially CohenMacaulay if and only if $\left.(G \backslash \mu)\right|_{\bar{S}}$ is sequentially Cohen-Macaulay for all $\mu \in \operatorname{Ind}(G)$ with $\mu \subseteq S$.

Proof. When $G$ is a bipartite graph, the graph $G \cup W(S)$ will also be bipartite for any $S \subseteq V$. In addition, any subgraph of the form $(G \backslash \mu)_{\bar{S}}$ will also be bipartite. 
So, Theorem 4.10 implies $G \cup W(S)$ is sequentially Cohen-Macaulay if and only if Ind $(G \cup W(S))$ is vertex decomposable. But, by Theorem 4.6, Ind $(G \cup W(S))$ is vertex decomposable if and only if Ind $(G \backslash \mu)_{\bar{S}}$ is vertex decomposable for all $\mu \in \operatorname{Ind}(G)$ with $\mu \subseteq S$. But, again by Theorem 4.10, this can happen if and only if $(G \backslash \mu)_{\bar{S}}$ is sequentially Cohen-Macaulay for all $\mu \in \operatorname{Ind}(G)$ with $\mu \subseteq S$.

Remark 4.12. It is natural to ask if Theorem 4.11 holds for all graphs, not just bipartite graphs. Examining the proof of Theorem 4.11, we might be able to find a counterexample if there exists a graph $G$ that is sequentially Cohen-Macaulay, but Ind $(G)$ is not vertex decomposable, i.e., if the converse of Theorem 4.3 is false. This is indeed the case. As pointed out in [8, Example 4.4], we can construct a graph $G$ from a minimal triangulation of the real projective plane such that $G$ is CohenMacaulay over a field $k$ if and only if the characteristic of $k$ is not 2 . In particular, over a field of characteristic $0, G$ is Cohen-Macaulay, but Ind $(G)$ is not vertex decomposable.

Acknowledgments. We used CoCoA [4], Macaulay2 [10] and the package SimplicialDecomposabilty by David Cook II [5] for our computer experiments.

\section{REFERENCES}

1. J. Biermann and A. Van Tuyl, Balanced vertex decomposable simplicial complexes and their h-vectors. Electr. J. Comb. 20 (2013) \#P15.

2. A. Björner, P. Frankl and R. Stanley, The number of faces of balanced CohenMacaulay complexes and a generalized Macaulay theorem, Combinatorica 7 (1987), $23-34$.

3. A. Björner and M. Wachs, Shellable nonpure complexes and posets I, Trans. Amer. Math. Soc. 348 (1996), 1299-1327.

4. CoCoATeam, CoCoA: A system for doing computations in commutative algebra, available at http://cocoa.dima.unige.it.

5. D. Cook, II, Simplicial decomposability, JSAG 2 (2010), 20-23.

6. D. Cook, II and U. Nagel, Cohen-Macaulay graphs and face vectors of flag complexes, SIAM J. Discr. Math. 26 (2012), 89-101.

7. A. Dochtermann and A. Engström, Algebraic properties of edge ideals via combinatorial topology, Electr. J. Combin. 16 (2009), Research Paper 2, 24 pages.

8. C. Francisco and H.T. Hà, Whiskers and sequentially Cohen-Macaulay graphs, J. Comb. Theor. 115 (2008), 304-316. 
9. A. Frohmader, How to construct a flag complex with a given face vector, preprint (2011) arXiv:1112.6061v1.

10. D.R. Grayson and M.E. Stillman, Macaulay2, A software system for research in algebraic geometry, http://www.math.uiuc.edu/Macaulay2/.

11. J. Herzog and T. Hibi, Monomial ideals, Grad. Texts Math. 260, SpringerVerlag, New York, 2011.

12. J. Jonsson, Simplicial complexes of graphs, Lect. Notes Math. 1928, Springer-Verlag, Berlin, 2008.

13. J. Provan and L. Billera, Decompositions of simplicial complexes related to the diameters of convex polyhedra, Math. Oper. Res. 5 (1980), 576-594.

14. A. Van Tuyl, Sequentially Cohen-Macaulay bipartite graphs: Vertex decomposability and regularity, Arch. Math. 93 (2009), 451-459.

15. R.H. Villarreal, Cohen-Macaulay graphs, Manuscr. Math. 66 (1990), 277293.

16. R. Woodroofe, Vertex decomposable graphs and obstructions to shellability, Proc. Amer. Math. Soc. 137 (2009), 3235-3246.

17. Chordal and sequentially Cohen-Macaulay clutters, Electr. J. Comb. 18 (2011), Paper 208, 20 pages.

Department of Mathematics and Statistics, 451A Clapp Lab, Mount Holyoke College, South Hadley, MA 01075

Email address: jbierman@mtholyoke.edu

Department of Mathematics, Oklahoma State University, 401 MathematiCal Sciences, Stillwater, OK 74078

Email address: chris.francisco@okstate.edu

Tulane University, Department of Mathematics, 6823 St. Charles Ave., New Orleans, LA 70118

Email address: tha@tulane.edu

Department of Mathematics and Statistics, McMaster University, HamilTON, ONTARIO L8S 4L8

Email address: vantuyl@math.mcmaster.ca 